\title{
Polymeric Side Chain Thioxanthone Photoinitiator for Free Radical Polymerization
}

\author{
Gokhan Temel $^{1}$, Nergis Arsu ${ }^{1}\left(\right.$ 冈), Yusuf Yagci $^{2}$ (凶) \\ ${ }^{1}$ Yildiz Technical University, Department of Chemistry, Davutpasa Campus, Istanbul 34210, \\ Turkey \\ ${ }^{2}$ Istanbul Technical University, Department of Chemistry, Maslak, Istanbul 34469, Turkey \\ E-mail: yusuf@itu.edu.tr; Fax: 902122856386
}

Received: 20 December 2005 / Revised version: 3 February 2006 / Accepted: 10 February 2006 Published online: 23 February 2006 - ( ) Springer-Verlag 2006

\begin{abstract}
Summary
Side chain thioxanthone-containing polymer (PSt-TX) was synthesized and characterized by modification of polystyrene prepared by Atom Transfer Radical Polymerization. PSt-TX exhibits absorption characteristics similar to that of the unsubstitued thioxanthone. Its capability to act as initiator for the polymerization of methyl methacrylate was also examined.
\end{abstract}

\section{Introduction}

Photoinitiated free radical polymerization is extensively used in numerous applications such as those in coatings, adhesives, inks, printing plates, optical waveguides and microelectronics [1-3]. Almost all of the commercially significant photoinitiators are organic compounds that produce free radicals upon irradiation [4]. The most widely used free radical photoinitiators include benzoin and derivatives, benzyl ketals, acetophenone derivatives, aromatic ketone / amine combinations. Among these initiators thioxanthone (TX) derivatives have recently received a revitalized interest because of their absorption characteristics at near UV range [5-13]. Moreover, their simple synthetic procedure allows various modifications for wavelength tunability or improved solubility by the incorporation appropriate substituents on the thioxanthone structure. Free radical generation process is $\mathrm{H}$ abstraction reaction of thioxanthone triplets from hydrogen donors such as amines and alcohols (Scheme 1). The radical derived from the donor can initiate the polymerization while the radicals stemming from TX are usually not reactive towards vinyl monomers due to bulkiness and/or the delocalization of the unpaired electron. Various structurally different thioxanthone derivatives including dendritic [5-6], polymeric and one-component ones [7-9] possessing both light absorbing chromophoric group and hydrogen donating sites in the same structure have been synthesized and their photochemistry has been studied in detail.

Polymeric photoinitiators have attracted much attention in the past years, for they combine properties of polymers with those of low molecular weight photoinitiators [14-15]. Solubility or miscibility problems, often observed with coatings containing low molecular weight photoinitators do not occur with the polymeric ones since 


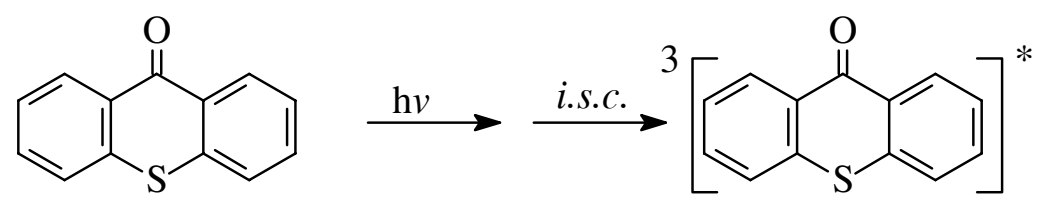

(TX)

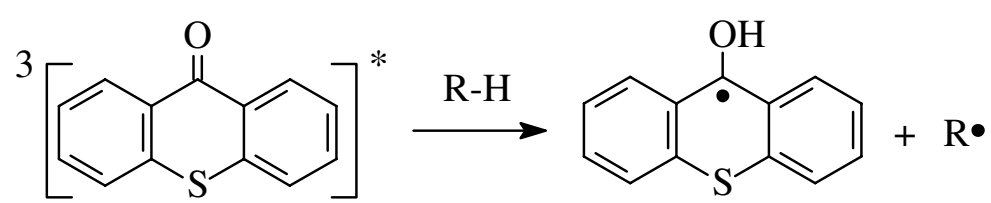

Scheme 1. Photoinduced radical generation from thioxanthone

polymers are easily miscible with the resin to be cured as well as with the final, cured film. Moreover, odor and toxicity problems do not occur with macrophotoinitiators owing to the low volatility of large molecules. The low migration tendency of polymeric photoinitators and of photoproducts brings about a reduced proneness to yellowing of cured coatings.

In this context, we synthesized a novel macrophotoinitiator through introducing thioxanthone moieties onto polystyrene prepared by Atom Transfer Radical Polymerization (ATRP). We also studied the photopolymerization of methyl methacrylate (MMA) by using the obtained photoinitiator.

\section{Experimental Section}

\section{Materials}

Thiosalicylic acid (98\%, Aldrich), $N$-methyldiethanolamine (MDEA; 99\%, Aldrich) were used as received. Styrene (99\%, Aldrich) and methyl methacrylate (99\%, Aldrich) were washed with $5 \%$ aqueous $\mathrm{NaOH}$ solution, dried over $\mathrm{CaCl}_{2}$, and distilled over $\mathrm{CaH}_{2}$ under vacuum just before use. Dimethylformamide (DMF; 99+\%, Aldrich) was distilled over $\mathrm{CaH}_{2}$ under reduced pressure. $\mathrm{N}, \mathrm{N}, \mathrm{N}, \mathrm{N}^{\prime}$ ', N' Pentamethyldiethylenetriamine (PMDETA, Aldrich) was distilled over $\mathrm{NaOH}$ before use. Tetrahydrofuran (99.8\%, J.T. Baker) was dried and distilled over $\mathrm{LiAlH}_{4}$. All other reagents were purchased from Aldrich and used as received.

\section{Instruments}

Gel permeation chromatography (GPC) analyses of the polymers were performed with a set-up consisting of a pump (Waters) and four ultrastyragel columns of different porosities. Tetrahydrofuran (THF) was used as the eluent (flow rate $3 \mathrm{~mL} \cdot \mathrm{min}^{-1}$ ), and the detection was carried out with the aid of a differential refractometer. The numberaverage molecular weights were determined using polystyrene standards. ${ }^{1} \mathrm{H}$ NMR spectra were recorded on a Bruker 250 instrument with d-DMSO as solvent and tetramethylsilane (TMS) as the internal standard. UV-visible spectra were taken on an Ati Unicam spectrophotometer. 


\section{Synthesis of polystyrene (PSt) by ATRP}

In a typical bulk polymerization, a Schlenk tube was charged with $3 \mathrm{~mL}$ (26.2 mmol) of styrene, $0.045 \mathrm{~g}(0.26 \mathrm{mmol})$ of PMDETA, $0.037 \mathrm{~g}(0.26 \mathrm{mmol})$ of CuBr. $0.051 \mathrm{~g}$ $(0.26 \mathrm{mmol})$ Ethyl-2-bromo- isobutyrate was added and the system was degassed three times. The tube was immersed in a preheated oil bath $\left(110{ }^{0} \mathrm{C}\right)$ for 16 minutes. The crude product was dissolved with THF, filtered with neutral alumina. Excess of THF was removed by rotary evaporater, and polymer solution was precipitated in 10fold excess methanol. The polymers were dried in vacuum at room temperature. The yields were determined gravimetrically.

\section{Preperation of polystyrene photoiniator with thioxanthone $(\underline{P S t-T X})$ moieties}

Thiosalycylic acid $1.7 \mathrm{~g}(11 \mathrm{mmol})$ was slowly added to $15 \mathrm{~mL}$ of concentrated sulfuric acid, and the mixture was stirred for $10 \mathrm{~min}$. PSt, $0.2 \mathrm{~g}(0.1 \mathrm{mmol})$, was added slowly to the stirred mixture over a period of $30 \mathrm{~min}$. After the addition, the reaction mixture was further stirred at room temperature for $1 \mathrm{~h}$ and then at $80{ }^{\circ} \mathrm{C}$ for $3 \mathrm{~h}$. At the end of this period, it was left to stand at room temperature overnight. The resulting mixture was poured with stirring into a 10-fold excess of boiling water, and it was boiled further for $5 \mathrm{~min}$. The solution was cooled and filtered. Yield: $70 \%$. $\mathrm{Mn}=4580$ $\mathrm{g} \mathrm{mol}^{-1}$ as determined by NMR and elemental analysis.

\section{Photopolymerization}

Typical procedure: Appropriate solutions of the monomer, MMA, and PSt-TX were irradiated in a photoreactor consisting of a $400 \mathrm{~W}$ medium pressure mercury lamp and a water cooling system, in an air atmosphere. Polymers were obtained after precipitation in methanol and drying under vacuum. Polymers were then purified by dissolving in THF and unreacted PSt-TX was readily separated as insoluble part. Dissolved PMMA was precipitated into methanol. Conversions were calculated gravimetrically.

\section{Results and Discussion}

Macrophotoinitiator containing side-chain TX moieties was synthesized by the modified literature procedure according to Scheme 2.<smiles>CC(C)(C)CC(c1ccccc1)C(C)(Br)[In]</smiles>

Scheme 2. Synthesis of polymeric thioxanthone photoinitiator 
ATRP is an established method to obtain various polymer architectures with predetermined molecular weights and narrow polydispersity [16]. ATRP involves reversible homolytic cleavage of a carbon-halogen bond by a redox reaction between an organic halide (R-X) and a transition metal, such as copper (I) salts. Polystyrene with low polydispersity $(M w / M n=1.04)$ was obtained by ATRP of styrene using ethyl-2-bromo-isobutyrate as initiator in the presence of the $\mathrm{CuBr} / \mathrm{PMDETA}$ complex. The polymerization condition and results are given in Table 1.

Table 1. ATRP of styrene at $110{ }^{\circ} \mathrm{C}$ initiated by ethyl-2-bromo-isobutyrate

\begin{tabular}{ccccc}
\hline Sample & $\begin{array}{c}\text { Time } \\
(\mathrm{min})\end{array}$ & $\begin{array}{c}\text { Conversion } \\
(\%)\end{array}$ & $\begin{array}{c}M_{n} \\
\left(\mathrm{~g} \mathrm{~mol}^{-1}\right)\end{array}$ & $M_{w} / M_{n}$ \\
\hline $\mathrm{PSt}-\mathrm{Br}$ & 16 & 12 & 1900 & 1.04 \\
\hline
\end{tabular}

${ }^{\mathrm{a}}[\mathrm{M}]_{0}:[\mathrm{I}]_{0}[\mathrm{CuBr}]:[\mathrm{PMDETA}]=100: 1: 1: 1$

The aromatic groups of the resulting polymer were then used as linkage units with which to form thioxanthone structures (Scheme 2). The structure was confirmed by FT-IR, ${ }^{1} \mathrm{H}-\mathrm{NMR}$ spectra and elemental analysis. The signal of carbonyl groups of PStTX is observed at $1629 \mathrm{~cm}^{-1}$ in FT-IR spectra. Moreover, compared with the precursor PSt, the appearance of the new signals related to the aromatic protons of the thioxanthone moiety in the ${ }^{1} \mathrm{H}-\mathrm{NMR}$ spectrum was considered as evidence of completion of the addition reaction (Figure 1).

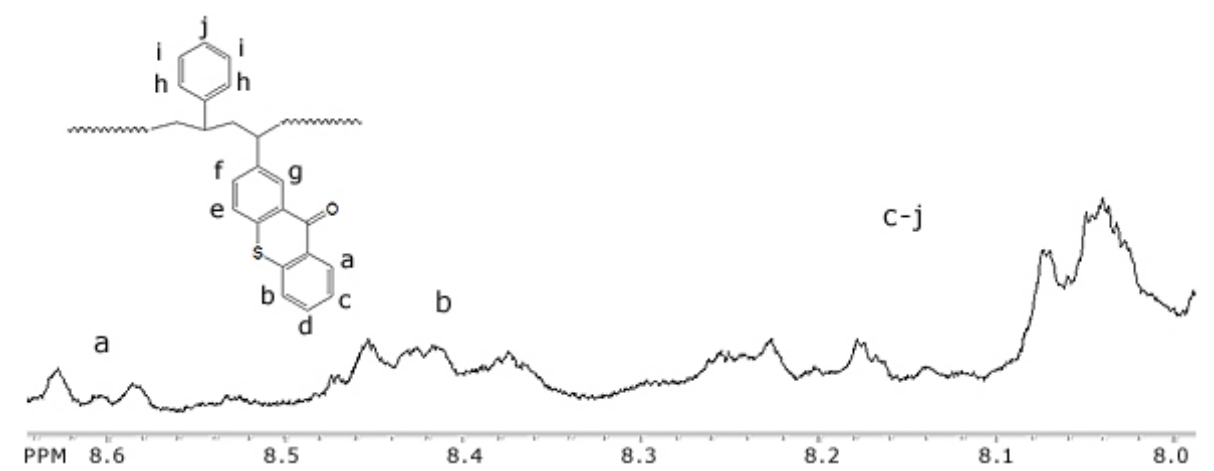

Figure 1. ${ }^{1} \mathrm{HNMR}$ spectra of PSt-TX in d-DMSO

The composition of PSt-TX was determined with the elemental analysis data (carbon content/sulfur content). The analysis results showed that about $80 \%$ aromatic groups of PSt reacted with thiosaliysilic acid. UV-vis absorption spectra of PSt-TX is shown in Figure 2a. It exhibits the usual characteristics absorption of thioxanthone with $\lambda_{\max }=389 \mathrm{~nm}$. Compared with the maximum of absorption of bare TX (Figure 2b), the observed slight shift may be attributed to the macromolecular structure of PSt-TX [12]. 


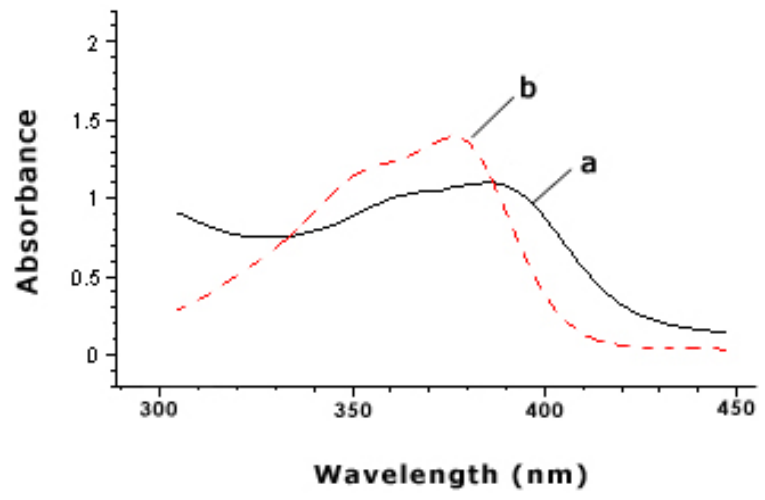

Figure 2. Optical absorption spectrum of PSt-TX a) and thioxanthone b) in presence of air in $\mathrm{DMF}$

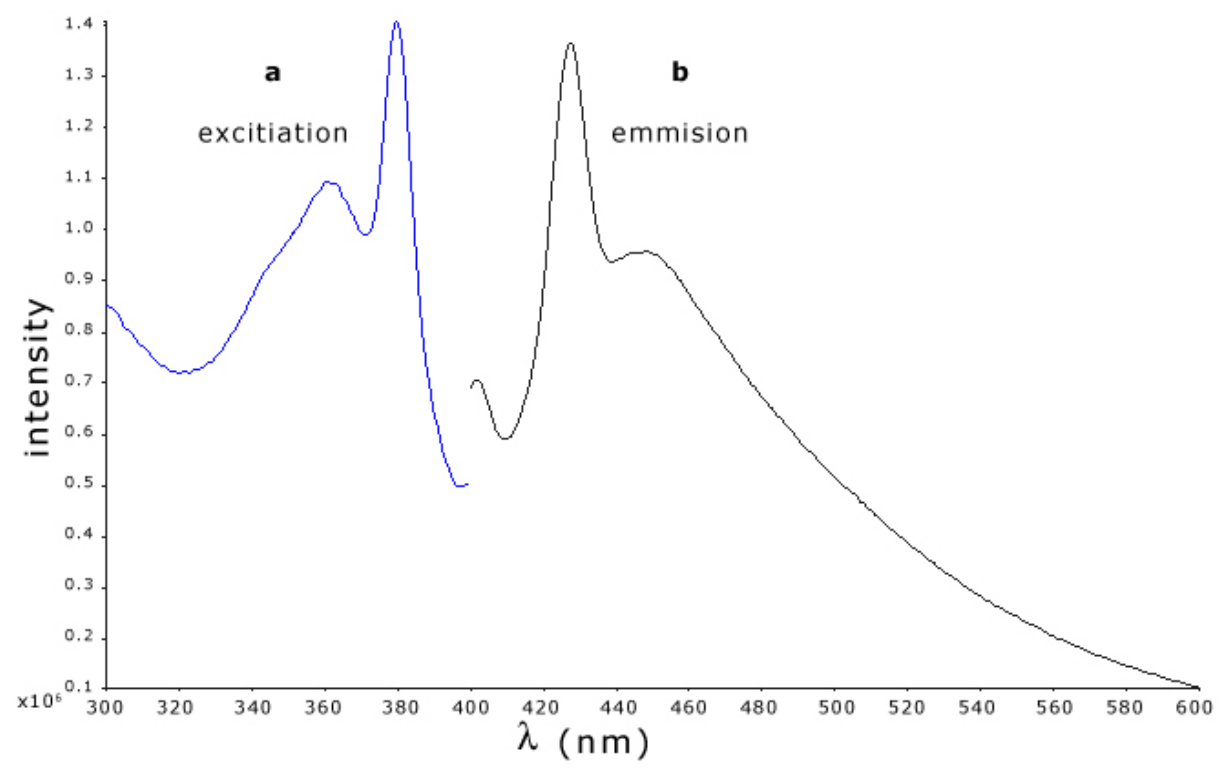

Figure 3. Fluorescence excitation (a) and emission spectrum (b) of PSt-TX in DMF at $25{ }^{\circ} \mathrm{C}$

Figure 3 shows that a nearly mirror-image-like relation exists between absorption and emission of PSt-TX again indicating successful incorporation of thioxanthone moieties. PSt-TX was used as a photoinitiator for the polymerization of MMA. The results are compiled in Table 2. The presence of an amine such as $\mathrm{N}$ methyldiethanolamine is important for effective photoreduction and photopolymerization (see Scheme 1, R-H = MDEA). It is also seen that the conversion is increased with increasing concentration of PSt-TX indicating that the rate of initiation is proportional to the absorbed light and consequently the amount of initiator. 
Table 2. Photoinitiated polymerization ${ }^{\mathrm{a}}$ of methyl methacrylate in DMF by using PSt-TX at room temp.

\begin{tabular}{cccc}
\hline $\begin{array}{c}{[\mathrm{PSt}-\mathrm{TX}]} \\
\left(\mathrm{mol} \mathrm{L}^{-1}\right)\end{array}$ & $\begin{array}{c}\text { Conversion } \\
(\%)\end{array}$ & $\begin{array}{c}M_{n}{ }^{\mathrm{b}} \times 10^{-4} \\
\left(\mathrm{~g} \mathrm{~mol}^{-1}\right)\end{array}$ & $M_{w} / M_{n}{ }^{\mathrm{b}}$ \\
\hline $5,1 \times 10^{-4}$ & 5,3 & 2,5 & 1,39 \\
$9,5 \times 10^{-4}$ & 8,8 & 3,1 & 1,50 \\
$1,9 \times 10^{-3}$ & 12,1 & 2,3 & 1,39 \\
$3,8 \times 10^{-3}$ & 14,1 & 2,0 & 1,45 \\
\hline
\end{tabular}

${ }^{\mathrm{a}}[\mathrm{MMA}]=4,68 \mathrm{~mol} \mathrm{~L}{ }^{-1},[\mathrm{MDEA}]=10^{-2} \mathrm{~mol} . \mathrm{L}^{-1}$, Time $=15 \mathrm{~min}$.

${ }^{\mathrm{b}}$ Determined by GPC according to PSt standards

\section{Conclusions}

In conclusion, although these results are preliminary in nature, they serve to indicate that polymeric side-chain thioxanthone photoinitiator can readily be synthesized by using polystyrene in the conventional synthetic procedure of of thioxanthones and used as an efficient photoinitiator for free radical polymerization. Studies on the photophysical and photochemical properties of the photoinitiator, together with the effords to prepare polymeric photoinitiators posseessing both thioxanthone and the hydrogen donating sites in the chain, are now in progress.

Acknowledgements. The authors thank Yildiz Technical University and Istanbul Technical University, Research Funds and Tubitak for their financial support.

\section{References}

1. Allen NS, Ed., (1987) Photopolymerization and Photoimaging Science and Technology Elsevier Applied Science, London

2. Fouassier JP, (1995) Photoinitiation, Photopolymerization, and Photocuring Hanser Verl., München

3. Davidson S, (1999) Exploring the Science, Technology and Applications of U.V. and E.B.Curing SITA Technology Ltd., London

4. Dietliker K, (1991) Chemistry \& Technology of UV \& EB Formulation for Coating, Inks \& Paints SITA Technology Ltd., London, Vol.3

5. Jiang, X, Yin J, (2004) Macromolecules 37:7850

6. Jiang X, Xu H, Yin J (2005) Polymer 46:11079

7. Aydin M, Arsu N, Yagci Y, (2003) Macromol. Rapid Commun. 24:718

8. Aydin M, Arsu N, Yagci Y, Jockusch S, Turro NJ, Macromolecules (2005) 38:4133

9. Cokbaglan L, Arsu N, Yagci Y, Jockusch S, Turro NJ (2003) Macromolecules 36:2649

10. Jiang X, Xu H, Yin J (2004) Polymer 45:133

11. Jiang $X$, Yin J, (2004) Polymer 45:5057

12. Jiang X, Yin J, (2004) Macromol. Rapid Commun. 25:748

13. Jiang X, Yin J, (2004) J. Appl. Polym. Sci. 94:2395

14. Davidson RS, (1993) J. Photochem. Photobiol. A: Chem. 69:263

15. Carlini C, Angiolini L, (1993) Radiat Curing Polym. Sci. Tech. 2:283

16. Matyjaszewski K, Xia J, Chem. Rev. (2001) 101:2921 